\title{
ON THE CONVERGENCE OF SOLUTIONS OF VOLTERRA EQUATIONS TO ALMOST-PERIODIC FUNCTIONS*
}

\author{
BY \\ GUSTAF GRIPENBERG
}

Helsinki University of Technology, SF-02150 Espoo 15

\begin{abstract}
The rate of convergence of solutions of a certain Volterra integral equation and a system of two Volterra equations to almost-periodic limit functions is studied. The equations considered arise from some diffusion problems with nonlinear and almostperiodic boundary conditions.
\end{abstract}

1. Introduction. The purpose of this paper is to study the asymptotic behavior of the solution $x$ of the nonlinear Volterra integral equation

$$
x(t)+\int_{0}^{t} a(t-s) g(s, x(s)) d s=f(t), \quad t \in R^{+}=[0, \infty),
$$

and we want to find an estimate for the rate of convergence of $x$ to an almost-periodic limit function. More generally, we also consider the system of equations

$$
\begin{aligned}
& x_{1}(t)+\int_{0}^{t} a_{1}(t-s) g_{1}\left(s, x_{1}(s)\right) d s+\int_{0}^{t} a_{2}(t-s) g_{2}\left(s, x_{2}(s)\right) d s=f_{1}(t), \\
& x_{2}(t)+\int_{0}^{t} a_{2}(t-s) g_{1}\left(s, x_{1}(s)\right) d s+\int_{0}^{t} a_{1}(t-s) g_{2}\left(s, x_{2}(s)\right) d s=f_{2}(t), \quad t \in R^{+} .
\end{aligned}
$$

The functions $f(t), f_{i}(t), g(t, y)$ and $g_{i}(t, y), i=1,2$, are assumed to be asymptotically almost periodic (as functions of $t$ ), and hence it is not surprising that one can, under certain conditions, prove that the same is true for the solutions $x(t)$ and $x_{i}(t), i=1,2$. This has been done in the special case $a(t)=(\pi t)^{-1 / 2}$ in [7], and in some cases when $a$ is integrable in [3] and [6]. The same question of asymptotic almost-periodicity for solutions of the system (1.2) is considered in [10] and in [11] the stability of the limit functions under small perturbations of the equations is established. In [12] and [13] some generalizations of this system of equations are studied using monotonicity properties of certain integral operators and basically the same ideas are used in this paper too. But here the main emphasis is not on the fact that the solutions converge to almost periodic limit functions (although this is shown under slightly weaker assumptions than before, i.e. we do not assume that $g(t, y)$ and $g_{i}(t, y)$ are locally Lipschitz continuous functions of $y$ ), but on the estimates for the rate of convergence.

Equations of the form (1.1) arise from the following diffusion problem: let $u$ be the

\footnotetext{
* Received May 22, 1980; revised version received August 10, 1980.
} 
solution

$$
\begin{gathered}
u_{t}=u_{y y}, \quad y>0, \quad t>0, \quad \lim _{y \rightarrow \infty} u(t, y)=0, \quad t>0, \\
u_{y}(t, 0)=g(t, u(t, 0), \quad t>0 .
\end{gathered}
$$

Then it follows (at least formally) that $x(t)=u(t, 0)$ satisfies $(1.1)$ with $a(t)=(\pi t)^{-1 / 2}$ and $f(t)=(2 \pi)^{-1} \int_{0}^{t}(t-s)^{-1 / 2} s^{-3 / 2} \int_{0}^{\infty} u(0, y) \exp \left(-y^{2} /(4 s)\right) d y d s, t \in R^{+}$.

The system (1.2) arises from a similar diffusion problem on a finite interval: Let $u$ be the solution of the equation $(L>0)$

$$
\begin{gathered}
u_{t}=u_{y y}, \quad 0<y<L, \quad t>0, \\
u_{y}(t, 0)=g_{1}(t, u(t, 0)), \quad u_{y}(t, L)=-g_{2}(t, u(t, L)), \quad t>0 .
\end{gathered}
$$

Then one gets the system (1.2) with $x_{1}(t)=u(t, 0), x_{2}(t)=u(t, L)$,

$$
a_{i}(t)=\pi L^{-1}\left(1+2 \sum_{n=1}^{\infty}(-1)^{(i-1) n} \exp \left(-(n \pi / L)^{2} t\right)\right), \quad i=1,2
$$

and

$$
\begin{aligned}
f_{i}(t)= & L^{-1} \int_{0}^{L} u(0, y) d y+\sum_{n=1}^{\infty} 2 L^{-1} \int_{0}^{L} u(0, y) \cos (n \pi y / L) d y \\
& \times(-1)^{(i-1) n} \exp \left(-(n \pi / L)^{2} t\right), \quad i=1,2, t \in R^{+} .
\end{aligned}
$$

The study of Eqs. (1.3) and (1.4) in [7] and [10] with $g(t, y)$ and $g_{i}(t, y), i=1,2$ of the form $c_{1}\left(y-c_{2} \sin (t)\right)^{3}$, was inspired by a theory of superfluidity of liquid helium (see [8]).

2. Statement of results. If $a_{2} \equiv 0$ in (1.2), then one has two equations of the form (1.1). Thus Eq. (1.1) is a special case of the system (1.2), but it turns out that the results one gets in this way from the theorem concerning system (1.2) are not the best possible ones. First we consider the more general, and harder, case of the system (1.2).

THEOREM 1. Assume that

$$
\begin{gathered}
a_{i} \in L_{\mathrm{loc}}^{1}\left(R^{+} ; R\right), \quad i=1,2, \\
A_{i} \in C^{\infty}((0, \infty)),(-1)^{k} A_{i}^{(k)}(t) \geq 0, \quad t>0, \quad k=0,1,2, \ldots, A_{i} \neq \equiv, 0, \\
i=1,2, \quad \text { where } A_{1}=a_{1}+a_{2}, A_{2}=a_{1}-a_{2}, \\
f_{i} \in C\left(R^{+} ; R\right), \quad i=1,2, \\
f_{i, \infty} \in C(R ; R) \text { is almost-periodic, } \quad i=1,2, \\
f_{i}(t)-f_{i, \infty}(t) \rightarrow 0 \quad \text { as } \quad t \rightarrow \infty, \quad i=1,2, \\
g_{i} \in C\left(R^{+} \times R ; R\right), \quad i=1,2,
\end{gathered}
$$

there exists a bounded function $p_{i}$ on $R^{+}$such that

$$
\left(y-p_{i}(t)\right) g_{i}(t, y) \geq 0, \quad t \in R^{+}, y \in R, \quad i=1,2 \text {, }
$$

there exists a positive number $\gamma_{0}$ such that if $|y| \geq \gamma_{0}$, then $\left|g_{i}\left(t, y+f_{i}(t)\right)\right| \geq \sup _{|z| \leq\left|f_{j}(t)-p_{j}(t)\right|+1}\left|g_{j}\left(t, z+f_{j}(t)\right)\right|, t \in R^{+}$, $i, j=1,2, \quad i \neq j$, 
$g_{i, \infty}(t, y) \in C(R \times R ; R)$ is an almost-periodic function of $t$, uniformly

for $y$ on compact subsets of $R, \quad i=1,2$,

$g_{i}(t, y)-g_{i, \infty}(t, y) \rightarrow 0$ as $t \rightarrow \infty$, uniformly for $y$ on compact

subsets of $R, i=1,2$,

there exists a continuous, nonnegative, increasing and convex

function $k$ on $R^{+}$such that $g_{i, \infty}(t, y)-g_{i, \infty}(t, z) \geq k(y-z)$,

$y>z, t \in R, \max \{|y|,|z|\} \leq 1+\gamma_{1}+\sup _{t \in R}\left|f_{i, \infty}(t)\right|, \quad i=1,2$,

where $\gamma_{1}=2 \max \left\{\gamma_{0}, \sup _{t \in R^{+}, i \in\{1,2\}}\left|f_{i}(t)-p_{i}(t)\right|\right\}$.

Then there exist continuous solutions $x_{1}$ and $x_{2}$ of (1.2) and continuous almost-periodic functions $x_{1, \infty}$ and $x_{2, \infty}$ such that

$$
\begin{aligned}
\left|x_{i}(t)-x_{i, \infty}(t)\right| & \leq\left|f_{i}(t)-f_{i, \infty}(t)\right|+2 k^{-1}\left(2 ^ { - 1 / 2 } t ^ { - 1 } \left(\int_{0}^{t} h(s) d s\right.\right. \\
& +3 \gamma_{1} \sum_{i=1}^{2}\left(A_{i}(1)^{-1}\right. \\
& \left.\left.\left.+\int_{1}^{t}\left(\left(\int_{0}^{s} A_{i}(\tau) d \tau\right)^{-1}-\left(\int_{0}^{\infty} A_{i}(\tau) d \tau\right)^{-1}\right) d s\right)\right)\right), \quad t \geq 1, i=1,2,
\end{aligned}
$$

where $k^{-1}$ is the inverse function of $k$ and

$$
h(t)=2^{1 / 2} \sup _{s \geq t,|y| \leq i 1, i \in\{1,2\}}\left|g_{i}\left(s, y+f_{i}(s)\right)-g_{i, \infty}\left(s, y+f_{i, \infty}(s)\right)\right| .
$$

For the properties of almost periodic functions that we will need, see e.g. [1].

The assumption (2.8) is a technical one that is used when one proves that the solutions of (1.2) are bounded, and it is satisfied if $g_{1}(t, y) \equiv g_{2}(t, y)$ is an odd and nondecreasing function of $y$ (cf. [10, Lemma 3]).

The claim in $\left[10\right.$, p. 566] that " $\hat{u}=(I-M(s) / N) u$ maps $S_{0}$ strictly inside $S$ " need not hold in general because the assumptions in [10, Lemma 5] do not exclude the possibility that $M(s)=0$. Thus it seems that some assumption of the form (2.11) would be needed for the proof in [10] to go through. This fact is also pointed out in the remarks following Lemma 2 of [13]. Concerning Eq. (1.1), we have the following result.

Theorem 2. Assume that

$$
\begin{aligned}
& a \in L_{\mathrm{loc}}^{1}\left(R^{+} ; R\right) \text { is positive, nonincreasing and } \log (a) \text { is convex on }(0, \infty), \\
& \qquad f \in C\left(R^{+} ; R\right), \\
& f_{\infty} \in C(R ; R) \text { is almost-periodic, } \\
& f(t)-f_{\infty}(t) \rightarrow 0 \text { as } t \rightarrow \infty, \\
& \quad g \in C\left(R^{+} \times R ; R\right) ;
\end{aligned}
$$

there exists a bounded function $p$ such that

$$
(y-p(t)) g(t, y) \geq 0, \quad t \in R^{+}, y \in R,
$$

$g_{\infty}(t, y) \in C\left(R^{+} \times R ; R\right)$ is an almost-periodic function of $t$, uniformly for $y$ on compact subsets of $R$,

$g(t, y)-g_{\infty}(t, y) \rightarrow 0$ as $t \rightarrow \infty$, uniformly for $y$ on compact subsets of $R$,

there exists a continuous, nonnegative and increasing function $k$ on $R^{+}$ such that $g_{\infty}(t, y)-g_{\infty}(t, z) \geq k(y-z), y>z, t \in R, \max \{|y|,|z|\} \leq$ $1+\sup _{t \in R^{+}}|f(t)-p(t)|+\sup _{t \in R^{+}}|f(t)|$. 
Then there exists a continuous solution $x$ of (1.1) and a continuous almost-periodic function $x_{\infty}$ such that

$$
\begin{aligned}
& \mid x(t)- x_{\infty}(t)|\leq| f(t)-f_{\infty}(t) \mid+k^{-1}\left(\operatorname { m a x } \left\{t^{-1} \int_{0}^{t} h(s) d s, \quad h(0)\right.\right. \\
&\left.\times \int_{t / 2}^{t} a(s) d s\left(\int_{0}^{t} a(s) d s\right)^{-1}+h(t / 2)\right\}+\left(1+2 \sup _{t \in R^{+}}|f(t)-p(t)|\right) \\
&\left.\times\left(\left(\int_{0}^{t} a(s) d s\right)^{-1}-\left(\int_{0}^{\infty} a(s) d s\right)^{-1}\right)\right), \quad t>0,
\end{aligned}
$$

where $k^{-1}$ is the inverse function of $k$ and

$$
h(t)=\sup _{s \geq t,|y| \leq \sup _{t, R^{+}} \mid f(\tau)-p(\tau)}\left|g(s, y+f(s))-g_{\infty}\left(s, y+f_{\infty}(s)\right)\right| .
$$

The proofs of these two theorems are similar to those in [10] and rely on a "variation of constants" equation and comparison arguments. Observe that we do not, as in $[3,7,10]$, assume that $g_{\infty}(t, y)$ and $g_{i, \infty}(t, y)$ are locally Lipschitz continuous functions of $y$.

3. Proof of Theorem 1. First we observe that we may without loss of generality assume that $g_{i}(t, y)$ and $g_{i, \infty}(t, y), i=1,2$, are locally Lipschitz continuous functions of $y$, with Lipschitz constant independent of $t$. If this is not already the case, then we replace $g_{i}$ by the function $2 m \int_{y-1 / m}^{y+1 / m} g_{i}(t, s) d s, t \in R^{+}, y \in R, i=1,2$ and similarly for $g_{i, \infty}$. To see that these functions are locally Lipschitz continuous we note that it follows from (2.9) that $g_{i, \infty}(t, y), i=1,2$, is uniformly bounded when $y$ is in a compact set (see [1, p. 52]), and by $(2.10)$ the same statement is true for the functions $g_{i}(t, y)$. All properties of $g_{i}$ and $g_{i, \infty}$ that we need remain unchanged (we may be forced to modify the functions $p_{i}$ slightly), and since none of our estimates depend on the Lipschitz constant we can let $m \rightarrow \infty$ at the end of our calculations. This is possible because the solutions we find will be uniformly bounded and equicontinuous and hence we can pick a subsequence that converges uniformly on compact sets.

Next we establish the existence and boundedness of a solution of the system (1.2) and first we reduce (exactly as in [10]) (1.2) to a system that is easier to handle. Let $R_{i, \lambda}$, $i=1,2, \lambda>0$, be the solutions of the equations

$$
R_{i, \lambda}(t)+\lambda^{-1} \int_{0}^{t} A_{i}(t-s) R_{i, \lambda}(s) d s=\lambda^{-1} A_{i}(t), \quad t \in R^{+} .
$$

It follows from (2.1), (2.2) and some results in [9, Sec. IV.7] that

$$
\begin{aligned}
& R_{i, \lambda} \in C^{\infty}((0, \infty)),(-1)^{k} R_{i, \lambda}^{(k)}(t) \geq 0, \quad t>0, k=0,1,2, \ldots, \\
& \int_{0}^{\infty} R_{i, \lambda}(t) d t=\left(\lambda\left(\int_{0}^{\infty} A_{i}(t) d t\right)^{-1}+1\right)^{-1}, \quad i=1,2, \lambda>0 .
\end{aligned}
$$

Let

$$
\begin{gathered}
Q=2^{-1 / 2}\left(\begin{array}{rr}
1 & 1 \\
1 & -1
\end{array}\right), \quad x(t)=\left(\begin{array}{l}
x_{1}(t) \\
x_{2}(t)
\end{array}\right), \quad f(t)=\left(\begin{array}{l}
f_{1}(t) \\
f_{2}(t)
\end{array}\right), \quad g(t, y)=\left(\begin{array}{l}
g_{1}\left(t, y_{1}\right) \\
g_{2}\left(t, y_{2}\right)
\end{array}\right), \\
R_{\dot{\lambda}}(t)=\left(\begin{array}{cc}
R_{1, \lambda}(t) & 0 \\
0 & R_{2, \lambda}(t)
\end{array}\right), \quad u(t)=Q(x(t)-f(t)), \quad t \in R^{+} .
\end{gathered}
$$

By (3.1), (3.3) and [10, Lemma 2] the system (1.2) can be transformed into

$$
u(t)=\int_{0}^{t} R_{\lambda}(t-s)(u(s)-\lambda Q g(s, Q u(s)+f(s))) d s, \quad t \in R^{+} .
$$

It follows from standard results (cf. [9, Chap. II]) that there exists a local solution of 
(3.4), hence also of (1.2), and in order to show that this solution can be extended to the whole of $R^{+}$it is sufficient to establish an a priori bound on the solution. Note also that $u$ is independent of $\lambda$ since $Q u+f$ is a solution of (1.2).

Let $\|y\|=\max \left\{\left|y_{1}\right|,\left|y_{2}\right|\right\}$ if $y \in R^{2}$. We deduce from (3.2), (3.4) and the proof of $[10$, Thm. 4] that

$$
\|u(t)\| \leq 2^{-1 / 2} \gamma_{1}, \quad t \in R^{+},
$$

provided we can show that if $\varepsilon>0$ is arbitrary and $\|y\| \leq 2^{-1 / 2}\left(\gamma_{1}+\varepsilon\right)$, then

$$
\sup _{t \in R^{+}}\|y-\lambda Q g(t, Q y+f(t))\| \leq 2^{-1 / 2}\left(\gamma_{1}+\varepsilon\right) \text {, }
$$

for some $\lambda>0$ (depending on $\varepsilon$ ). To do this we recall that we already noted above that $g(t, y)$ is uniformly bounded for $y$ on compact subsets of $R$. Hence we can choose $\lambda>0$ so that

$$
\lambda \sup _{\|z\| \leq ; 1+\varepsilon}\|g(t, z+f(t))\| \leq 2^{-1} \varepsilon, \quad t \in R^{+}
$$

The absolute value of the first component of $y-\lambda Q g(t, y+f(t))$ has the upper bound (add and subtract $y_{2} / 2$ )

$$
\begin{aligned}
\left|\left(y_{1}+y_{2}\right) 2^{-1}-\lambda 2^{-1 / 2} g_{1}\left(t,\left(y_{1}+y_{2}\right) 2^{-1 / 2}+f_{1}(t)\right)\right| & \\
& +\left|\left(y_{1}-y_{2}\right) 2^{-1}-\lambda 2^{-1 / 2} g_{2}\left(t,\left(y_{1}-y_{2}\right) 2^{-1 / 2}+f_{2}(t)\right)\right| .
\end{aligned}
$$

If we use the same estimate for the second component and apply (2.7), (2.8), (3.7), the definition of $\gamma_{1}$ (in (2.11)) and the assumption that $\|y\| \leq 2^{-1 / 2}\left(\gamma_{1}+\varepsilon\right)$, then we obtain (3.6). Thus we conclude that (3.5) holds.

The assumptions (2.4) and (2.9) imply that there exists a sequence $\left\{t_{n}\right\}_{n=1}^{\infty}$ tending to $\infty$ such that

$$
g_{i, \infty}\left(t+t_{n}, y\right) \rightarrow g_{i, \infty}(t, y), \quad f_{i, \infty}\left(t+t_{n}\right) \rightarrow f_{i, \infty}(t) \quad \text { as } n \rightarrow \infty, \quad i=1,2,
$$

uniformly on $R$, and also uniformly for $y$ on compact subsets of $R$. It follows from (3.2), (3.4), (3.5) and the uniform boundedness of $g(t, y)$ that $u$ is uniformly continuous and therefore there exists a uniformly continuous and bounded function $v\left(\|v(t)\| \leq 2^{-1 / 2} \gamma_{1}\right)$ and a subsequence of $\left\{t_{n}\right\}$, again denoted by $\left\{t_{n}\right\}$, such that $u\left(t+t_{n}\right) \rightarrow v(t)$ as $n \rightarrow \infty$, uniformly on compact subsets of $R$. Now it follows from (2.5), (2.10), (3.2), (3.4) and (3.8) that

$$
v(t)=\int_{-\infty}^{t} R_{\lambda}(t-s)\left(v(s)-\lambda Q g_{\infty}\left(s, Q v(s)+f_{\infty}(s)\right) d s, \quad t \in R .\right.
$$

Here $g_{\infty}(t, y)=\left(g_{1, \infty}\left(t, y_{1}\right), g_{2, \infty}\left(t, y_{2}\right)\right)^{T}$ and $f_{\infty}(t)=\left(f_{1, \infty}(t), f_{2, \infty}(t)\right)^{T}$. We observe that since $u$ is independent of $\lambda$, so is $v$ also.

Next we will try to find an upper bound for $\|u(t)-v(t)\|$, and it also remains for us to show that $v$ is almost periodic.

By (3.4) and (3.9) we have

$$
\begin{aligned}
u(t)-v(t)= & \int_{0}^{t} R_{\dot{\lambda}}(t-s)\left(u(s)-v(s)-\lambda Q\left(g_{\infty}\left(s, Q u(s)+f_{\infty}(s)\right)-g_{\infty}(s, Q v(s)\right.\right. \\
& \left.\left.\left.+f_{\infty}(s)\right)\right)-\lambda Q\left(g(s, Q u(s)+f(s))-g_{\infty}\left(s, Q u(s)+f_{\infty}(s)\right)\right)\right) d s \\
& -\int_{-\infty}^{0} R_{\lambda}(t-s)\left(v(s)-\lambda Q g_{\infty}\left(s, Q v(s)+f_{\infty}(s)\right)\right) d s, \quad t \in R^{+} .
\end{aligned}
$$


We choose $\lambda$ to be sufficiently small, compared to the Lipschitz constant of $g_{\infty}$, and we want to show that

$$
\begin{aligned}
\left\|u(t)-v(t)-\lambda Q\left(g_{\infty}\left(t, Q u(t)+f_{\infty}(t)\right)-g_{\infty}\left(t, Q v(t)+f_{\infty}(t)\right)\right)\right\| & \\
& \leq\|u(t)-v(t)\|-\lambda K(\|u(t)-v(t)\|), \quad t \in R^{+}
\end{aligned}
$$

where

$$
K(s)=2^{1 / 2} k\left(s 2^{-1 / 2}\right), \quad s \in R^{+} .
$$

To derive the inequality (3.11) we break up the first component of the vector on the left-hand side into two terms; the first term can, by (2.11) and the assumption that $g_{1, \infty}$ is locally Lipschitz continuous, be estimated as follows:

$$
\begin{array}{r}
\mid\left(u_{1}(t)-v_{1}(t)+u_{2}(t)-v_{2}(t)\right) 2^{-1}-\lambda 2^{-1 / 2}\left(g_{1, \infty}\left(t,\left(u_{1}(t)+u_{2}(t)\right) 2^{-1 / 2}+f_{1, \infty}(t)\right)\right. \\
\left.-g_{1, \infty}\left(t,\left(v_{1}(t)+v_{2}(t)\right) 2^{-1 / 2}+f_{1, \infty}(t)\right)\right)|\leq| u_{1}(t)-v_{1}(t)+u_{2}(t)-v_{2}(t) \mid 2^{-1} \\
-\lambda 2^{-1 / 2} k\left(\left|u_{1}(t)-v_{1}(t)+u_{2}(t)-v_{2}(t)\right| 2^{-1 / 2}\right) .
\end{array}
$$

The other terms are treated in the same manner and if we add the two terms that come from the same component and use the definition (3.12), then we get (3.11). Note that here we use the fact that $k$ is convex, because then $\left(k\left(\left|y_{1}-y_{2}\right|\right)+k\left(\left|y_{1}+y_{2}\right|\right)\right) 2^{-1} \geq k(\|y\|)$.

Define the functions $m, w_{1}, w_{2}$ and the constant $c_{i}$ by (we will later use the fact that $c_{\lambda}$ is sufficiently large with respect to the bound on $u$ )

$$
\begin{aligned}
w_{i}(t) & =\left|u_{i}(t)-v_{i}(t)\right|, i=1,2, \quad m(t)=\max \left\{w_{1}(t), w_{2}(t)\right\}, \quad t \in R^{+}, \\
c_{\lambda} & =\max \left\{\sup _{t \leq 0}\left\|v(t)-\lambda Q g_{\infty}\left(t, Q v(t)+f_{\infty}(t)\right)\right\|, 3 \gamma_{1}\right\} .
\end{aligned}
$$

From (3.2), (3.10), (3.11) and (3.13) it follows that (recall that $h$ is defined in the statement of Theorem 1)

$$
w_{i}(t) \leq \int_{0}^{t} R_{i, \lambda}(t-s)(m(s)-\lambda K(m(s))+\lambda h(s)) d s+c_{\lambda} \int_{t}^{\infty} R_{i, \lambda}(s) d s, \quad t \in R^{+}, i=1,2 .
$$

If $\lambda$ is sufficiently small, then the function: $s \rightarrow s-\lambda K(s)$ is monotone increasing for the values of $s$ that we are interested in, and therefore we can apply standard comparison arguments to the inequalities above (see [9, Sec. II.6]). We conclude that there exist solutions $W_{i}$ of the equations

$$
\begin{aligned}
W_{i}(t)= & \int_{0}^{t} R_{i, \lambda}(t-s)(M(s)-\lambda K(M(s)) \\
& +\lambda h(s)) d s+c_{\lambda} \int_{t}^{\infty} R_{i, \lambda}(s) d s, \quad t \in R^{+}, i=1,2,
\end{aligned}
$$

where $M(t)=\max \left\{W_{1}(t), W_{2}(t)\right\}$, such that

$$
w_{i}(t) \leq W_{i}(t), \quad t \in R^{+}, i=1,2 .
$$

These functions $W_{i}$ are not necessarily independent of $\lambda$, but this fact is of no consequence. 
We want to replace $R_{i, \lambda}$ in (3.14) by one function $r_{\lambda}$ with certain desirable properties so that

$$
W_{i}(t) \leq \int_{0}^{t} r_{\lambda}(t-s)(M(s)-\lambda K(M(s))+\lambda h(s)) d s+c_{\lambda} \int_{t}^{\infty} r_{\lambda}(s) d s, \quad t \in R^{+}, i=1,2 .
$$

Before we can do this we must show that the functions $W_{i}$ (and hence also $M$ ) are absolutely continuous and nonincreasing. Observe that by (3.2) and (3.14), $W_{i}(0)=$ $c_{\lambda}\left(\lambda\left(\int_{0}^{\infty} A_{i}(s) d s\right)^{-1}+1\right)^{-1}$; we then deduce, from our bounds on $\|u(t)\|$ and $\|v(t)\|$ and the definition of $c_{\lambda}$, that we may without loss of generality assume that $K$ is such that $K(M(0))>h(0)+c_{\lambda}\left(\int_{0}^{\infty} A_{i}(s) d s\right)^{-1}$, for all small $\lambda$ and $i=1$, 2. If we now solve Eqs. (3.14) by iteration, and initially substitute $W_{i}(t) \equiv c_{\lambda} \int_{0}^{\infty} R_{i, \lambda}(s) d s$ on the right-hand side, then we see, since $h$ is nonincreasing, $R_{i, \lambda}(t) \geq 0$ and $K(M(0))$ satisfies the inequality above, that all iterates are absolutely continuous and nonincreasing. We conclude that the same assertion is true for the functions $W_{i}$.

Now we proceed to define the function $r_{i}$. Let the functions $B_{i}, i=1,2$, be defined as the solutions of the equations

$$
A_{i}(t) / A_{i}(0)+\int_{0}^{t} B_{i}(t-s) A_{i}(s) d s=1, \quad t>0, i=1,2 .
$$

Here $1 / A_{i}(0)=\lim _{t \rightarrow 0+} 1 / A_{i}(t)$. Next we define the function $b$ by

$$
b(t)=B_{1}(t)-B_{1}(\infty)+B_{2}(t)-B_{2}(\infty), \quad t \in R^{+}
$$

$\left(B_{i}(\infty)=\lim _{t \rightarrow \infty} B_{i}(t)\right)$; we take $1 / a(0)=1 / A_{1}(0)+1 / A_{2}(0)$ and let $a$ be the solution of the equation

$$
a(t) / a(0)+\int_{0}^{t} b(t-s) a(s) d s=1, \quad t>0 .
$$

Finally the function $r_{\lambda}, \lambda>0$, is defined to be the solution of the equation

$$
r_{\lambda}(t)+\lambda^{-1} \int_{0}^{t} a(t-s) r_{\lambda}(s) d s=\lambda^{-1} a(t), \quad t \in R^{+} .
$$

It follows from (2.1), (2.2) and (3.17)-(3.20) that all the functions $B_{i}, i=1,2, b, a$ and $r_{\lambda}$, $\lambda>0$, are locally integrable and completely monotone (i.e. $C^{\infty}$, with nonnegative even derivatives and nonpositive odd ones). To see this one can take Laplace transforms of Eqs. (3.17)-(3.20) and apply [4, Thm. 4] or [5, Thm. 9.1, pp. 236-238] (recall that a function is completely monotone if and only if it is of the form $\int_{0}^{\infty} e^{-s t} d \mu(s)$, where $\mu$ is a nonnegative measure).

Next we are going to establish (3.16), and for this reason we solve $M(t)-$ $\lambda K(M(t))+\lambda h(t)$ from (3.14) with the aid of (3.17); we deduce from some calculations, in which (3.1) and (3.2) are used, that

$$
\begin{aligned}
& M(t)-\lambda K(M(t))+\lambda h(t)=W_{i}(t)+\lambda W_{i}^{\prime}(t) / A_{i}(0) \\
& \quad+\lambda \int_{0}^{t}\left(B_{i}(t-s)-B_{i}(\infty)\right) W_{i}^{\prime}(s) d s+B_{i}(\infty) W_{i}(t), \text { a.e. } t \in R^{+}, \quad i=1,2 .
\end{aligned}
$$


Since the functions $W_{i}$ are nonnegative and nonincreasing, it follows from (3.18) and our definition of $a(0)$ that

$$
\begin{aligned}
& M(t)-\lambda K(M(t))+\lambda h(t) \geq W_{i}(t)+\lambda W_{i}^{\prime}(t) / a(0) \\
& +\lambda \int_{0}^{t} b(t-s) W_{i}^{\prime}(s) d s, \text { a.e. } t \in R^{+}, i=1,2 .
\end{aligned}
$$

From this inequality we conclude, since $r_{\lambda}(t) \geq 0$ and $b(\infty)=0$, that (3.16) holds (we go through the same calculations as above but now we use (3.19), (3.20) and the fact that $\left.W_{i}(0) \leq c_{j}\right)$.

As an immediate consequence of (3.16) and the definition of $M$ we have

$$
M(t) \leq \int_{0}^{t} r_{\lambda}(t-s)(M(s)-\lambda K(M(s))+\lambda h(s)) d s+c_{\lambda} \int_{t}^{\infty} r_{\lambda}(s) d s, \quad t \in R^{+},
$$

and this inequality implies by $(3.20)$ that

$$
M(t)+\int_{0}^{t} a(t-s) K(M(s)) d s \leq \int_{0}^{t} a(t-s) h(s) d s+c_{i}, \quad t \in R^{+} .
$$

Note that $b(\infty)=0$ implies that $\int_{0}^{\infty} a(s) d s=\infty$. Since $M$ is a nonincreasing and $K$ an increasing nonnegative function we have

$$
K(M(t)) \int_{0}^{t} a(s) d s \leq M(t)+\int_{0}^{t} a(t-s) K(M(s)) d s, \quad t \in R^{+} .
$$

If we recall that $h$ and $a$ are nonnegative and nonincreasing, then we can deduce from a lemma due to Tchebysheff (see [2, p. 278]) that

$$
\int_{0}^{t} a(t-s) h(s) d s \leq t^{-1} \int_{0}^{t} h(s) d s \int_{0}^{t} a(s) d s, \quad t \in R^{+} .
$$

Next we establish a lower bound for $\int_{0}^{t} a(s) d s$. Using the fact that $a$ and $b$ are nonnegative, we see after integrating (3.19) over $(0, t)$ that

$$
\int_{0}^{t} a(s) d s \geq t\left(1 / a(0)+\int_{0}^{t} b(s) d s\right)^{-1}, \quad t>0 .
$$

If we invoke the fact that both $A_{i}$ and $B_{i}$ are nonnegative and nonincreasing, then we get from (3.17) that

$$
\begin{gathered}
B_{i}(t)-B_{i}(\infty) \leq\left(\int_{0}^{t} A_{i}(s) d s\right)^{-1}-\left(\int_{0}^{\infty} A_{i}(s) d s\right)^{-1}, \quad t>0, \\
1 / A_{i}(0)+\int_{0}^{1} B_{i}(s) d s \leq 1 / A_{i}(1), \quad i=1,2 .
\end{gathered}
$$

If we combine these three inequalities with (3.18) and the definition of $1 / a(0)$, then we obtain

$$
\int_{0}^{t} a(s) d s \geq t\left(\sum_{i=1}^{2}\left(1 / A_{i}(1)+\int_{1}^{t}\left(\left(\int_{0}^{s} A_{i}(\tau) d \tau\right)^{-1}-\left(\int_{0}^{\infty} A_{i}(\tau) d \tau\right)^{-1}\right) d s\right)\right)^{-1}, \quad t>1 .
$$


To prove that (2.12) holds (with $\left.\left(x_{1, \infty}(t), x_{2, \infty}(t)\right)^{T}=Q v(t)+f_{\infty}(t)\right)$, we let $\lambda \rightarrow 0$ and use the definition of $u$ in (3.3) and also (3.12), (3.13), (3.15) and (3.21)-(3.24). Therefore it remains for us to prove that the function $v$ is almost-periodic.

If we proceed in the same way as in the proof of [10, Thm. 6], we conclude that if $v$ is not almost-periodic, then there exist a number $\varepsilon>0$, two sequences $\left\{t_{i, k}\right\}_{k=1}^{\infty}$ and vector functions $g_{0}(t, y), f_{0}(t), v_{*}(t)$ and $v_{* *}(t)\left(g_{0}=\left(g_{1,0}, g_{2,0}\right)^{T}\right.$ and $\left.f_{0}=\left(f_{1,0}, f_{2,0}\right)^{T}\right)$, such that

$$
\begin{gathered}
\left\|v_{*}(0)-v_{* *}(0)\right\| \geq \varepsilon, \\
g_{\infty}\left(t+t_{i, k}, y+f_{\infty}\left(t+t_{i, k}\right)\right) \rightarrow g_{0}\left(t, y+f_{0}(t)\right) \text { as } k \rightarrow \infty, \quad i=1,2,
\end{gathered}
$$

uniformly on $R$ and uniformly for $y$ on compact subsets of $R$, and

$$
v\left(t+t_{1, k}\right) \rightarrow v_{*}(t), \quad v\left(t+t_{2, k}\right) \rightarrow v_{* *}(t) \quad \text { as } \quad k \rightarrow \infty,
$$

uniformly on compact subsets of $R$. It follows from (3.9), (3.26) and (3.27) that

$$
\begin{aligned}
v_{*}(t)-v_{* *}(t)= & \int_{-\infty}^{t} R_{\lambda}(t-s)\left(v_{*}(s)-\lambda Q g_{0}\left(s, Q v_{*}(s)+f_{0}(s)\right)\right. \\
& \left.-v_{* *}(s)+\lambda Q g_{0}\left(s, Q v_{* *}(s)+f_{0}(s)\right)\right) d s, \quad t \in R .
\end{aligned}
$$

We have to show that if $\lambda$ is sufficiently small, then

$$
\begin{aligned}
&\left\|v_{*}(t)-\lambda Q g_{0}\left(t, Q v_{*}(t)+f_{0}(t)\right)-v_{* *}(t)+\lambda Q g_{0}\left(t, Q v_{* *}(t)+f_{0}(t)\right)\right\| \\
& \leq \max \left\{\varepsilon / 2,\left\|v_{*}(t)-v_{* *}(t)\right\|-\lambda 2^{-1} K\left(\left\|v_{*}(t)-v_{* *}(t)\right\|\right)\right\}, \quad t \in R .
\end{aligned}
$$

The functions $g_{i, 0}(t, y)$ satisfy $(2.11)$ (with $g_{i, \infty}$ replaced by $g_{i, 0}$ ) since (3.26) holds, and are uniformly continuous for $y$ on compact subsets of $R$ (see [1, p. 52]) but not necessarily locally Lipschitz continuous as functions of $y$. We must study expressions of the form $\left(Q_{i} y\right.$ is the $i$ th component of $Q y$ if $\left.y \in R^{2}\right)$,

$$
\sum_{i=1}^{2} 2^{-1 / 2}\left|Q_{i}\left(v_{*}(t)-v_{* *}(t)\right)-\lambda\left(g_{i, 0}\left(t, Q_{i} v_{*}(t)+f_{i, 0}(t)\right)-g_{i, 0}\left(t, Q_{i} v_{* *}(t)+f_{i, 0}(t)\right)\right)\right|,
$$

and we can choose a number $\delta>0$ so that

$$
\left|g_{i, 0}\left(t, Q_{i} v_{*}(t)+f_{i, 0}(t)\right)-g_{i, o}\left(t, Q_{i} v_{* *}(t)+f_{i, o}(t)\right)\right| \leq 2^{-1} k(\varepsilon / 8)-k(\delta),
$$

if $\left|Q_{i} v_{*}(t)-Q_{i} v_{* *}(t)\right| \leq \delta$. If now $\lambda$ is sufficiently small, then

$$
\begin{aligned}
\mid Q_{i}\left(v_{*}(t)-v_{* *}(t)\right)-\lambda\left(g_{i, 0}(\right. & \left.t, Q_{i} v_{*}(t)+f_{i, o}(t)\right)-g_{i, o}\left(t, Q_{i} v_{* *}(t)\right. \\
& \left.\left.+f_{i, 0}(t)\right)\right)|\leq| Q_{i}\left(v_{*}(t)-v_{* *}(t)\right) \mid-\lambda k\left(\left|Q_{i}\left(v_{*}(t)-v_{* *}(t)\right)\right|\right)
\end{aligned}
$$

if $\left|Q_{i}\left(v_{*}(t)-v_{* *}(t)\right)\right| \geq \delta$. If we use these observations, then we see from a simple argument that (3.29) holds if $\lambda$ is sufficiently small. Since (3.2), (3.25), (3.28) and (3.29) imply a contradiction, we conclude that $v$ must be almost-periodic and the proof of Theorem 1 is completed.

4. Proof of Theorem 2. The proof of Theorem 2 follows closely that of Theorem 1, but is simpler because we do not have to work with vector-valued functions and the matrix $Q$.

For the same reasons as above we may assume that $g_{\infty}(t, y)$ is a locally Lipschitz 
continuous function of $y$. Eq. (1.1) is transformed into

$$
u(t)=\int_{0}^{t} r_{\lambda}(t-s)(u(s)-\lambda g(s, u(s)+f(s))) d s, \quad t \in R^{+},
$$

where $u(t)=x(t)-f(t)$ and $r_{\lambda}$ is defined by (3.20) (but now we do not have (3.17)-(3.19)). It follows from (2.13) (see [9, Sec. IV.6]) that

$$
r_{\lambda}(t) \geq 0, \quad t \in R^{+}, \quad \int_{0}^{\infty} r_{\lambda}(t) d t=\left(\lambda\left(\int_{0}^{\infty} a(t) d t\right)^{-1}+1\right)^{-1}, \quad \lambda>0 .
$$

In order to show that

$$
|u(t)| \leq \sup _{t \in R^{+}}|f(t)-p(t)|,
$$

we need by (4.2) only note that it follows from (2.18) that if $\varepsilon>0$ is arbitrary and $|y| \leq \sup _{t \in R^{+}}|f(t)-p(t)|+\varepsilon$, then $|y-\lambda g(t, y+f(t))| \leq \sup _{t \in R^{+}}|f(t)-p(t)|+\varepsilon$, provided $\lambda$ is small enough.

Exactly as in the proof of Theorem 1, we obtain the existence of a function $v$ such that

$$
v(t)=\int_{-\infty}^{t} r_{\lambda}(t-s)\left(v(s)-\lambda g_{\infty}\left(s, v(s)+f_{\infty}(s)\right)\right) d s, \quad t \in R .
$$

It follows from (2.21) and (4.1)-(4.3) that

$$
\begin{aligned}
|u(t)-v(t)| \leq \int_{0}^{t} r_{\lambda}(t-s)(|u(s)-v(s)|-\lambda k(\mid u(s) & -v(s) \mid) \\
& +\lambda h(s)) d s+c_{\lambda} \int_{t}^{\infty} r_{\lambda}(s) d s, \quad t \in R^{+},
\end{aligned}
$$

where $c_{\lambda}=\max \left\{\sup _{t \leq 0}\left|v(t)-\lambda g_{\infty}\left(t, v(t)+f_{\infty}(t)\right)\right|, 2 \sup _{t \in R^{+}}|f(t)-p(t)|+1\right\}$. Using a standard comparison theorem, we deduce that there exists a function $M$, seen to be nonincreasing by the same argument that was used in the proof of Theorem 1 , such that

$$
\begin{aligned}
|u(t)-v(t)| \leq M(t)=\int_{0}^{t} r_{\lambda}(t-s)(M(s)-\lambda k(M(s)) & +\lambda h(s)) d s \\
& +c_{i} \int_{t}^{\infty} r_{i}(s) d s, \quad t \in R^{+} .
\end{aligned}
$$

Now it follows from (3.20) that

$$
\begin{aligned}
M(t)+\int_{0}^{t} a(t-s) k(M(s)) d s=\int_{0}^{t} a(t-s) h(s) d s+c_{\lambda}( & 1-\left(\lambda+\int_{0}^{t} a(s) d s\right) \\
& \left.\times\left(\lambda+\int_{0}^{\infty} a(s) d s\right)^{-1}\right), \quad t \in R^{+} .
\end{aligned}
$$

To establish (2.22) (with $x_{\infty}=v+f_{\infty}$ ), we have now only to note that $k(M(t)$ ) is a nonincreasing function of $t$ and that $\int_{0}^{t} a(t-s) h(s) d s \leq h(0) \int_{t / 2}^{t} a(s) d s+$ $h(t / 2) \int_{0}^{t / 2} a(s) d s$, and then proceed in the same way as in the proof of Theorem 1. To prove that $v$ is almost-periodic and thus complete the proof of Theorem 2, we argue in the same manner as in the proof of Theorem 1. 


\section{REFERENCES}

[1] C. Corduneanu, Almost periodic functions, Interscience Publishers, New York, 1968

[2] W. F. Donoghue, Distributions and Fourier transforms, Academic Press, New York, 1969

[3] A. Friedman, Periodic behavior of solutions of Volterra integral equations, J. Analyse Math. 15, 287-303 (1965)

[4] G. Gripenberg, On Volterra equations of the first kind, Integ. Eqs. Operator Th. (to appear)

[5] I. I. Hirschman and D. V. Widder, The convolution transform, Princeton University Press, Princeton, 1955

[6] M. J. Leitman and V. J. Mizel, Asymptotic stability and the periodic solutions of $x(t)+$ $\int_{-\infty}^{t} a(t-s) g(s, x(s)) d s=f(t)$, J. Math. Anal. Appl. 66, 606-625 (1978)

[7] N. Levinson, A nonlinear Volterra equation arising in the theory of superfluidity, J. Math. Anal. Appl. 1, $1-11(1960)$

[8] C. C. Lin, Hydrodynamics of liquid helium II, Phys. Rev. Letters 2, 245-246 (1959)

[9] R. K. Miller, Nonlinear Volterra integral equations, W. A. Benjamin, Menlo Park, 1971

[10] R. K. Miller, Almost periodic behavior of solutions of a nonlinear Volterra system, Quart. Appl. Math. 28, 553-570 (1971)

[11] R. K. Miller, Asymptotically almost periodic solutions of a nonlinear Volterra system, SIAM J. Math. Anal. 2, 435-444 (1971)

[12] D. G. Weis, Asymptotic behavior of some nonlinear Volterra integral equations, J. Math. Anal. Appl. 49, 59-87 (1975)

[13] D. G. Weis, Asymptotic behavior of integral equations using monotonicity, J. Math. Anal. Appl. 54, 49-58 (1976) 